\title{
PageRank analysis reveals topologically expressed genes correspond to psoriasis and their functions are associated with apoptosis resistance
}

\author{
XUE ZENG $^{1}$, JINGJING ZHAO ${ }^{2}$, XIAOHONG WU ${ }^{1}$, HONGBO SHI $^{2}$, WALI LIU ${ }^{1}$,

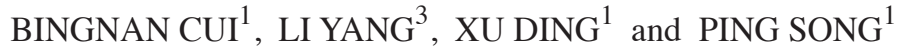 \\ ${ }^{1}$ Department of Dermatology, Guang'anmen Hospital, China Academy of Chinese Medical Sciences, Beijing 201203;
${ }^{2}$ Key Laboratory of Advanced Control and Optimization for Chemical Processes of the Ministry of Education,
East China University of Science and Technology, Shanghai 200237; ${ }^{3}$ Department of Otorhinolaryngology,
Guang'anmen Hospital, China Academy of Chinese Medical Sciences, Beijing 201203, P.R. China
}

Received April 24, 2015; Accepted February 19, 2016

DOI: $10.3892 / \mathrm{mmr} .2016 .4999$

\begin{abstract}
Psoriasis is an inflammatory skin disease. Deceleration in keratinocyte apoptosis is the most significant pathological change observed in psoriasis. To detect a meaningful correlation between the genes and gene functions associated with the mechanism underlying psoriasis, 927 differentially expressed genes (DEGs) were identified using the Gene Expression Omnibus database, GSE13355 [false discovery rate $(F D R)<0.01$; llog fold change $>1$ ] with the package in $\mathrm{R}$ langue. The selected DEGs were further constructed using the search tool for the retrieval of interacting genes, in order to analyze the interaction network between the DEGs. Subsequent to PageRank analysis, 14 topological hub genes were identified, and the functions and pathways in the hub genes network were analyzed. The top-ranked hub gene,
\end{abstract}

Correspondence to: Mrs. Ping Song, Department of Dermatology, Guang'anmen Hospital, China Academy of Chinese Medical Sciences, 5 Bei Xian'ge Street, Xicheng, Beijing 201203, P.R. China E-mail: songping@vip.126.com

Abbreviations: ER $\alpha$, estrogen receptor $\alpha$; DEGs, differentially expressed genes; STRING, search tool for the retrieval of interacting genes; GEO, gene expression omnibus database; GO, gene ontology; KEGG, Kyoto Encyclopedia of Genes and Genomes; ESR1, estrogen receptor-1; MCL1, myeloid cell leukemia-1; BIRC5, baculoviral IAP repeat containing-5; MMP9, matrix metalloproteinase-9; IL-1B, interleukin-1 $\beta$; IL-8, interleukin-8; APOE, apolipoprotein E; CCDN1, cyclin D1; BCL-2, B-cell lymphoma-2; BAX, BCL2-associated X protein; STAT1, signal transducer and activator of transcription-1; STAT3, signal transducer and activator of transcription-3; FOS, FBJ murine osteosarcoma viral oncogene homolog; CXCL10, chemokine (C-X-C motif) ligand 10; E2, $17 \beta$-estradiol; TLRs, toll-like receptors; NF- $\kappa \mathrm{B}$, nuclear factor- $\kappa \mathrm{B}$

Key words: psoriasis, differentially expressed genes, PageRank analysis, estrogen receptor-1, resistance of apoptosis estrogen receptor-1 (ESR1) is downregulated in psoriasis, exhibited binding sites enriched with genes possessing anti-apoptotic functions. The ESRl gene encodes estrogen receptor $\alpha(\mathrm{ER} \alpha)$; a reduced level of $\mathrm{ER} \alpha$ expression provides a crucial foundation in response to the anti-apoptotic activity of psoriatic keratinocytes by activating the expression of anti-apoptotic genes. Furthermore, it was detected that the pathway that is associated most significantly with psoriasis is the pathways in cancer. Pathways in cancer may protect psoriatic cells from apoptosis by inhibition of ESRl expression. The present study provides support towards the investigation of ESR 1 gene function and elucidates that the interaction with anti-apoptotic genes is involved in the underlying biological mechanisms of resistance to apoptosis in psoriasis. However, further investigation is required to confirm the present results.

\section{Introduction}

Psoriasis is an inherent and inflammatory skin disease with scaly erythematous lesions, affecting 2-3\% of the Caucasian population (1). The scales are a result of a hyperproliferative epidermis, as well as parakeratosis, and the highly mitotic rate of psoriatic keratinocytes results in a thickened epidermis. Complex pathogenic mechanisms associated with genetic expression variances and different immune responses result in psoriasis aggravation (2).

In addition to hyperproliferation, the psoriatic keratinocytes exhibit an abnormal resistance to apoptosis. Deceleration in keratinocyte apoptosis is the most significant pathological change in psoriasis (3). The decrease in apoptosis, which causes imbalances in skin homeostasis, may be associated with the induction of psoriatic hyperplasia (3). Multiple factors have been identified to contribute to anti-apoptosis in psoriatic keratinocytes, including the activation of mitogenic signaling pathways and the inactivation of certain apoptotic molecules $(4,5)$. In the present study, various PageRank hub genes were identified to be associated with anti-apoptosis, as well as with the regulation of apoptosis in psoriasis. Furthermore, the current study identified that the target genes in anti-apoptosis 
and apoptosis regulation are tightly associated with the ESRI gene. The ESR 1 gene encodes an estrogen receptor $\alpha(E R \alpha)$. The estrogen stimulates a rapid relocation of $\mathrm{ER} \alpha$ to induce high mitochondrial gene expression (6). It has been clearly demonstrated that activation of the mitochondrial signaling pathway, as well as the extrinsic apoptosis signaling pathway, is key in the process of many forms of programmed cell death (5). Thus, it was hypothesized in the present study that $\mathrm{ER} \alpha$ may exert a protective role against psoriatic proliferation.

The cytokine related Toll-like receptor (TLR) signaling pathway, a classical signaling pathway, is considered to be important in the progression of psoriasis (7). However, based on the analyses of the present study, the pathways in cancer was the most significant pathway that correlated with psoriasis, demonstrating a more significant association than the TLR signaling pathway. Pathways in cancer affect tumor proliferation by protecting the tumor from cytokine-induced apoptosis. The current results suggest that the activation of pathways in cancer may inhibit psoriatic keratinocyte apoptosis.

To analyze the disease-associated genes and gene functions, the differentially expressed genes (DEGs) associated with psoriasis were screened and compared with healthy controls from the DNA microarray database. A gene interaction network was generated for topological analysis of relevant genes associated with psoriasis using PageRank and the gene enrichment functions were subsequently evaluated. The top-ranked PageRank hub gene, ESRl, which is downregulated in psoriasis, exhibited binding sites enriched with anti-apoptotic genes that provides support for the negative association between estrogen and apoptosis. The current results demonstrate that reduced levels of ER $\alpha$ expression result in the anti-apoptotic behavior of psoriatic keratinocytes by activating anti-apoptotic gene expression. Pathways in cancer may protect the psoriatic cell from apoptosis by inhibiting ESRI expression. The present study provides evidence regarding ESRl gene function and demonstrates that the interaction with anti-apoptotic genes is involved in the underlying biological mechanisms of apoptosis resistance in psoriasis.

\section{Materials and methods}

Affymetrixmicroarraydataandpreprocessing. The microarray gene expression profiles were extracted from the Gene Expression Omnibus (GEO) database (GEO accession no. GSE13355; http://www.ncbi.nlm.nih.gov/geo/query/acc.cgi?acc=GSE13355) $(8,9)$, which contained gene expression profiles for 58 psoriatic patients and 64 healthy control subjects. All samples were run on GeneChip ${ }^{\circledR}$ Human Genome U133 Plus 2.0 Array (Affymetrix, Inc., Santa Clara, CA, USA), microarrays containing $>54,000$ gene probes. The raw data were processed using the Robust Multichip Average (RMA) method (5). The expression values were adjusted using RMA expression values (on the log scale) to account for batch and gender effects.

DEG identification. DEGs between psoriasis and normal skin were identified using the limma R package (10). The raw P-value was corrected by the Benjamin and Hochberg method to circumvent the multi-test bias (11). Finally, the false discovery rate $(F D R)<0.01$ and $\mid l o g$ fold change $(F C) \mid>1$ were used as the cut-off for DEG identification.
Interaction network construction of DEGs. A gene interaction network was generated by mapping all the DEGs in the Search Tool for the Retrieval of Interacting Genes (STRING) database, which is a database for predicting functional associations between proteins (12). The interactions include direct (physical) and indirect (functional) associations, and all of the associations are derived from four sources: Genomic context, high-throughput experiments, coexpression (conserved) and previous knowledge (13). All of the associating pairs were selected based on a combined probabilistic confidence score.

Identification of top-ranked hub genes using Google PageRank score. In order to identify hub genes (key genes), the PageRank score was calculated in all DEGs. The PageRank analysis was initially applied to Google (the web search engine) to identifying significant web pages (14-16) and has been used during the robust analysis of protein networks to identify important nodes (17). Unlike simply calculating the degree of each gene, the PageRank score measures the importance or popularity of a gene based solely on the interaction (link) structure of the interaction network. It selects the genes that exhibit a high degree, whilst also maintaining the important low-degree genes, which link to other important genes in the protein-protein interaction network. It is expected that the genes with more interaction links, especially those from important genes, are given higher PageRank scores.

Gene-annotation enrichment analysis using the Database for Annotation, Visualization and Integrated Discovery (David). DAVID software (Laboratory of Human Retrovirology and Immunoinformatics, Clinical Services Program; Leidos Biomedical Research, Inc., Frederick, MD, USA) $(18,19)$, a gene-annotation enrichment analysis tool, adopts a common core strategy to systematically map a hubs gene list to the associated biological annotation [for example, gene ontology (GO) terms or Kyoto Encyclopedia of Genes and Genomes (KEGG) pathways], and then statistically highlight the most overrepresented (enriched) biological annotation out of thousands of linked terms and contents. In the present analysis, GO and KEGG pathway annotations were integrated to evaluate the function enrichment of the hub genes.

Statistical analysis. DAVID software $(18,19)$ was used for all statistical analyses, and GO terms and KEGG pathways were selected using a standard parameter setting (FDR $<0.05$ and $\mathrm{P}<0.1)$. All the known human genes were used as the background for Fisher's exact test, and a P-value was calculated and the Bonferroni correction was applied.

\section{Results}

$D E G$ analysis. After extracting the gene expression data from the public dataset, GSE13355, the DEGs between psoriasis lesions and normal skin were identified. DEGs $(n=927 ; 449$ upregulated and 478 downregulated) were selected (FDR $<0.01$ and $\log \mathrm{FCl}>1)$ and a heat map of the DEG expression level between the two groups is presented in Fig. 1.

Construction and topological analysis of an interaction network. The interaction network was constructed using STRING and 


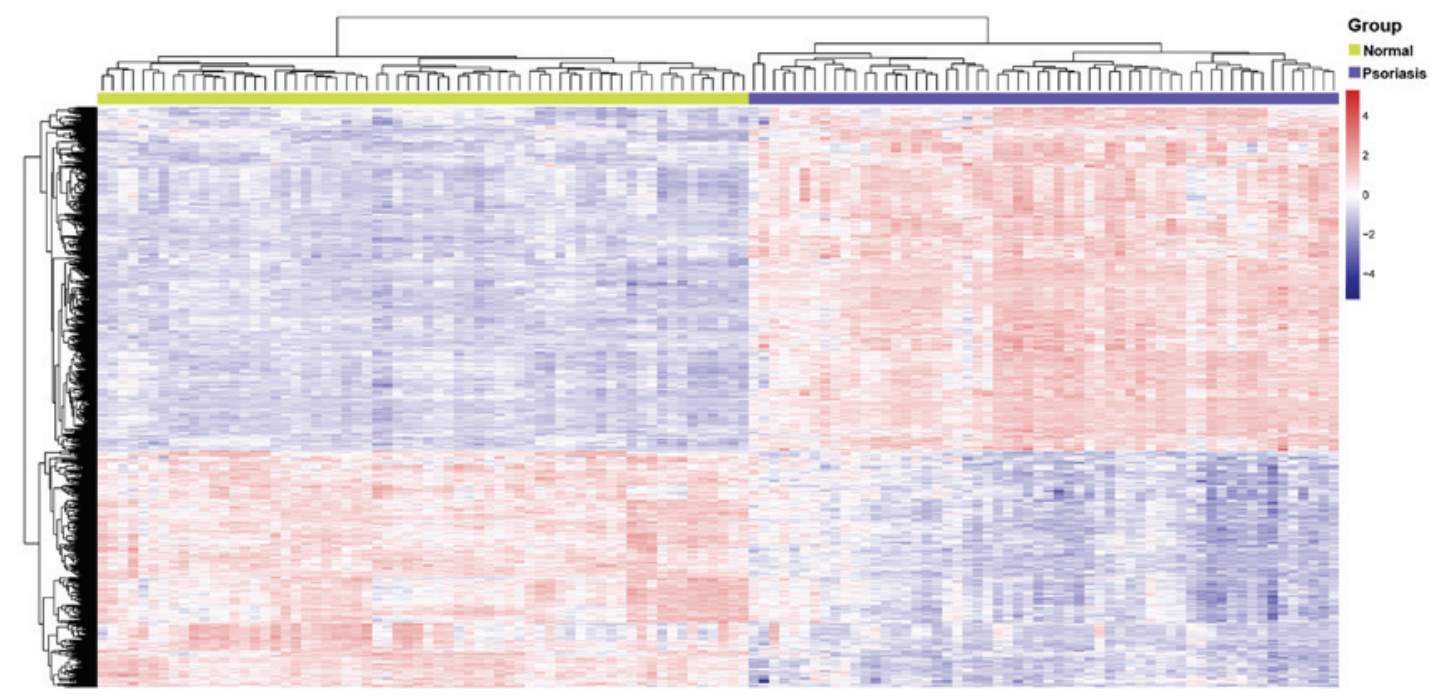

Figure 1. Heat map of the expression level of differentially expressed genes between psoriasis lesions and normal skin.

Table I. PageRank, degree distribution and the FC of the top 14 nodes in the interaction network.

\begin{tabular}{llcr}
\hline Gene name & Page-rank score & $\begin{array}{c}\text { Degree } \\
\text { distribution }\end{array}$ & $\operatorname{logFC}$ \\
\hline ESR1 & 0.007521607 & 56 & -1.149321612 \\
IL-1B & 0.007016809 & 55 & 1.097904425 \\
STAT1 & 0.006989954 & 75 & 2.906284146 \\
IL-8 & 0.006069015 & 53 & 4.192048124 \\
CXCL10 & 0.00604473 & 69 & 2.828564993 \\
FOS & 0.006031463 & 48 & -1.125965959 \\
MMP9 & 0.005700515 & 47 & 1.565431608 \\
STAT3 & 0.00500142 & 44 & 1.102377065 \\
APOE & 0.004996554 & 33 & -1.088425518 \\
CCND1 & 0.0048065 & 42 & -1.00144553 \\
COMP & 0.00457533 & 23 & 1.397789198 \\
MCL1 & 0.004510315 & 24 & 1.207643197 \\
LPL & 0.004289353 & 26 & -1.162938926 \\
BIRC5 & 0.004181229 & 86 & 1.746154734 \\
\hline
\end{tabular}

FC, fold change; ESR1, estrogen receptor-1; IL-1B, interleukin-1 $\beta$; STAT1, signal transducer and activator of transcription-1; IL-8, interleukin-8; CXCL10, chemokine (C-X-C motif) ligand 10; FOS, FBJ murine osteosarcoma viral oncogene homolog; MMP9, matrix metalloproteinase-9; STAT3, signal transducer and activator of transcription-3; APOE, apolipoprotein E; CCDN1, cyclin D1; COMP, cartilage oligomeric matrix protein; MCL1, myeloid cell leukemia-1; LPL, lipoprotein lipase; BIRC5, baculoviral IAP repeat containing-5.

the DEGs that had been identified. In total, 640 genes were identified and the interaction network of the DEGs is presented in Fig. 2. Based on the network topological analysis, it was found that the protein interaction network exhibited scale-free attributes. Three network topologies were processed using statistical analyses and are presented in Fig. 3. Fig. 3A shows the node degree distribution in the network, the $\mathrm{x}$-axis represents the node degrees (the number of direct connections the node has with other nodes) and the y-axis represents the number of nodes with the different degrees. The node degree distribution indicates that both loosely and closely connected nodes exist in the network. Fig. 3B demonstrates the clustering coefficient distribution in the network, and the clustering coefficient displays the aggregation degree of nodes; the distribution range of the clustering coefficient is between 0 and 1. Fig. $3 \mathrm{C}$ shows the path length of the network, indicating that the predominant path length was $\sim 3-4$. Subsequent to the analysis of the three topological property parameters of the interaction network, the present study was able to demonstrate the small-world effect of the network, which is subject to scale-free property (20).

PageRank hub genes and enrichment analysis. PageRank analysis was adopted to select the most popular and important genes within the interaction network. A high PageRank score is associated with a high degree. According to analysis of the PageRank score, 14 genes with the highest PageRank scores 


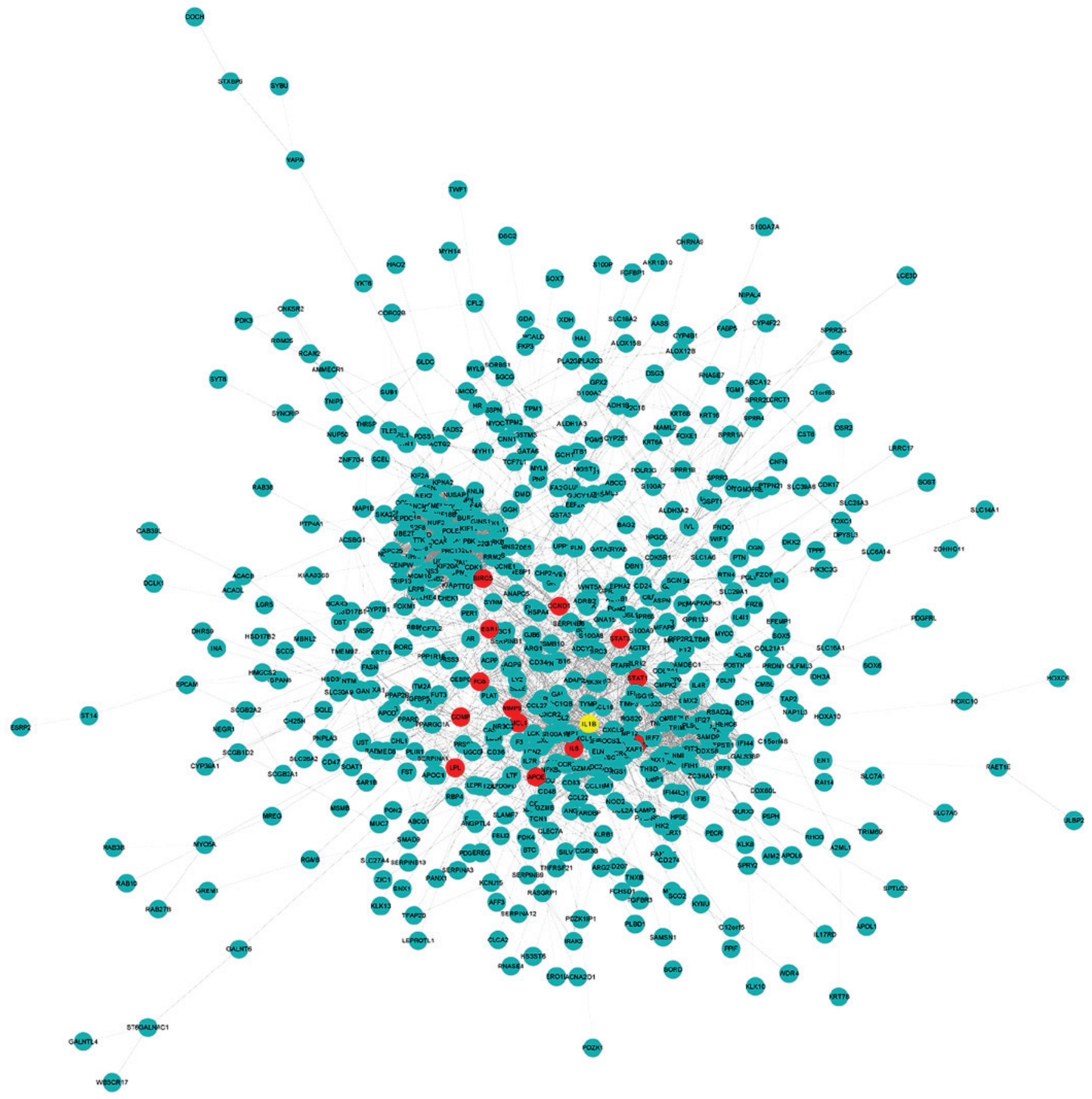

Figure 2. Interaction network of the differentially expressed genes. The yellow-colored node is the top hub gene, ESRI, with highest PageRank score. The 13 nodes highlighted red are the hub genes with the next highest PageRank scores, including IL-1B, STAT1, IL-8, CXCL10, FOS, MMP9, STAT3, APOE, CCND1, COMP, MCL1, LPL and BIRC5. IL-1B, interleukin-1ß; STAT1, signal transducer and activator of transcription-1; IL-8, interleukin-8; CXCL10, chemokine (C-X-C motif) ligand 10; FOS, FBJ murine osteosarcoma viral oncogene homolog; MMP9, matrix metalloproteinase-9; STAT3, signal transducer and activator of transcription-3; APOE, apolipoprotein E; CCDN1, cyclin D1; COMP, cartilage oligomeric matrix protein; MCL1, myeloid cell leukemia-1; LPL, lipoprotein lipase; BIRC5, baculoviral IAP repeat containing-5.

were selected as the top hub genes (Table I). Thirteen of the top 14 hub genes are highlighted in red and ESRI, identified as the top hub gene, was highlighted in yellow in the interaction network (Fig. 2). ESRI, representing high relativity with psoriasis, demonstrates a downregulated level of expression in Table I. The other 13 of the top hub genes, demonstrating various levels of expression, are presented in Table I.

To gain further insight into the functions of the top 14 hub genes, the enrichment of GO functions and KEGG pathway analysis were performed using the DAVID tool. The significant GO enrichment terms are presented in Table II. The function of anti-apoptosis was confirmed to be highly associated with psoriasis. Six of the top hub genes [ESR], apolipoprotein E (APOE), myeloid cell leukemia-1 (MCL1), cartilage oligomeric matrix protein $(C O M P)$, baculoviral IAP repeat containing-5 $(B I R C 5)$ and interleukin-1 $\beta(I L-1 B)]$ were identified to be involved in the anti-apoptosis effects of psoriasis (Table II). The other functions, such as regulation of apoptosis, programmed 
Table II. Gene ontology enrichment terms in the top-ranked 14 hub genes.

\begin{tabular}{|c|c|c|c|}
\hline Term & Count & FDR & Genes \\
\hline Anti-apoptosis & 6 & 0.001376778 & MCL1, APOE, COMP, ESR1, IL-1B, BIRC5 \\
\hline Response to organic substance & 8 & 0.002326258 & FOS, CCND1, MCL1, APOE, ESR1, IL-1B, STAT1, STAT3 \\
\hline Regulation of apoptosis & 8 & 0.004836793 & MCL1, APOE, MMP9, COMP, ESR1, IL-1B, BIRC5, STAT1 \\
\hline Regulation of programmed cell death & 8 & 0.005168359 & MCL1, APOE, MMP9, COMP, ESR1, IL-1B, BIRC5, STAT1 \\
\hline Regulation of cell death & 8 & 0.005297534 & MCL1, APOE, MMP9, COMP, ESR1, IL-1B, BIRC5, STAT1 \\
\hline Response to organic cyclic substance & 5 & 0.006196132 & FOS, CCND1, IL-1B, STAT1, STAT3 \\
\hline Negative regulation of apoptosis & 6 & 0.019556803 & MCL1, APOE, COMP, ESR1, IL-1B, BIRC5 \\
\hline $\begin{array}{l}\text { Negative regulation of programmed } \\
\text { cell death }\end{array}$ & 6 & 0.020933116 & $M C L 1, A P O E, C O M P, E S R 1, I L-1 B, B I R C 5$ \\
\hline Negative regulation of cell death & 6 & 0.021217271 & MCL1, APOE, COMP, ESR1, IL-1B, BIRC5 \\
\hline Pathways in cancer & 7 & 0.022194793 & FOS, CCND1, IL-8, MMP9, BIRC5, STAT1, STAT3 \\
\hline Response to hormone stimulus & 6 & 0.023292142 & FOS, CCND1, ESR1, IL-1B, STAT1, STAT3 \\
\hline Toll-like receptor signaling pathway & 6 & 0.030280341 & FOS, CCND1, MCL1, ESR1, BIRC5, STAT1 \\
\hline Response to endogenous stimulus & 6 & 0.03749322 & FOS, CCND1, ESR1, IL-1B, STAT1, STAT3 \\
\hline Response to steroid hormone stimulus & 5 & 0.038520418 & FOS, CCND1, ESR1, IL-1B, STAT3 \\
\hline
\end{tabular}

FDR, false discovery rate; MCL1, myeloid cell leukemia-1; APOE, apolipoprotein E; COMP, cartilage oligomeric matrix protein; ESR1, estrogen receptor-1; IL-1B, interleukin-1 $\beta$; BIRC5, baculoviral IAP repeat containing-5; FOS, FBJ murine osteosarcoma viral oncogene homolog; CCDN1, cyclin D1; MCL1, myeloid cell leukemia-1; STAT1, signal transducer and activator of transcription-1; STAT3, signal transducer and activator of transcription-3; MMP9, matrix metalloproteinase-9; IL-8, interleukin-8.
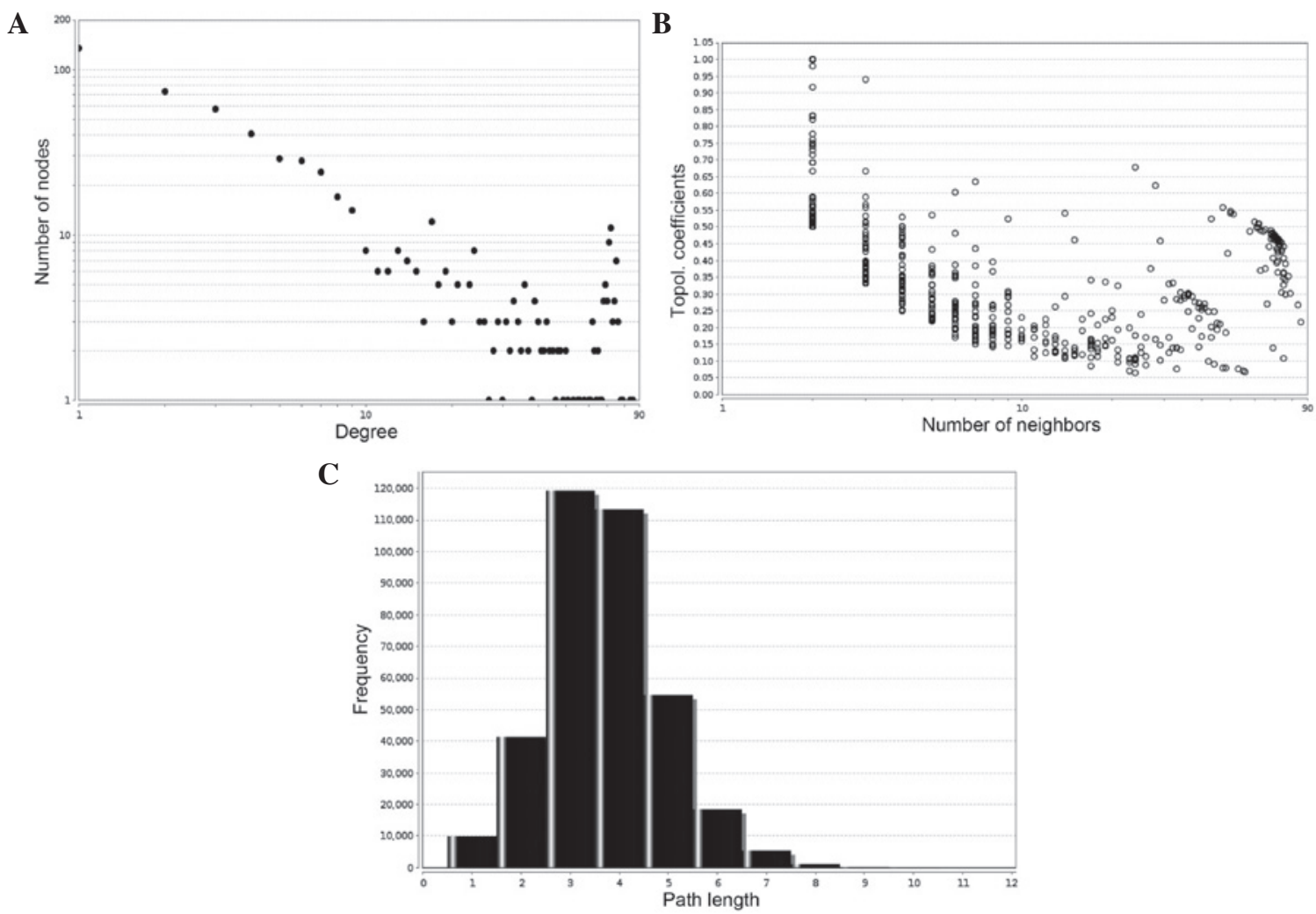

Figure 3. Three topological parameters of the interaction network of differentially expressed genes. Distribution graphs of (A) node degree, (B) clustering coefficient and (C) path length.

cell death and cell death, also represent the association with psoriasis. The same eight top hub genes [ESR1, APOE, MCL1, COMP, BIRC5, IL-1B, matrix metalloproteinase-9 (MMP9) and signal transducer and activator of transcription-1 (STAT1)] were observed to be correlated with regulation of apoptosis, programmed cell death and cell death (Table II). 


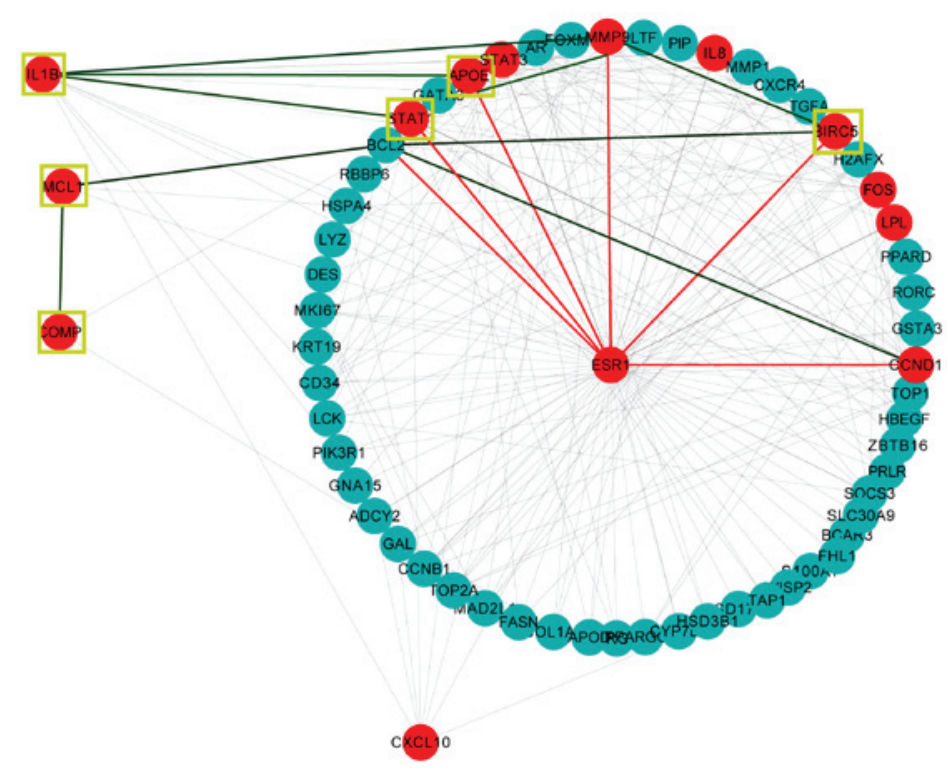

Figure 4. ESRI topological neighbors. The 56 nodes form a circle as they form ESRI topological neighbors. The 14 nodes that are highlighted in red are the top hub genes that are associated with psoriasis, and include the ESR1 gene. The yellow square represents the anti-apoptosis gene ontology annotation node. The red line links genes to the ESR1 gene and the green line links genes to the neighbors of the ESR1 gene. ESR1, estrogen receptor-1; IL-1B, interleukin-1 STAT1, signal transducer and activator of transcription-1; IL-8, interleukin-8; CXCL10, chemokine (C-X-C motif) ligand 10; FOS, FBJ murine osteosarcoma viral oncogene homolog; MMP9, matrix metalloproteinase-9; STAT3, signal transducer and activator of transcription-3; APOE, apolipoprotein E; CCDN1, cyclin D1; COMP, cartilage oligomeric matrix protein; MCL1, myeloid cell leukemia-1; LPL, lipoprotein lipase; BIRC5, baculoviral IAP repeat containing-5.

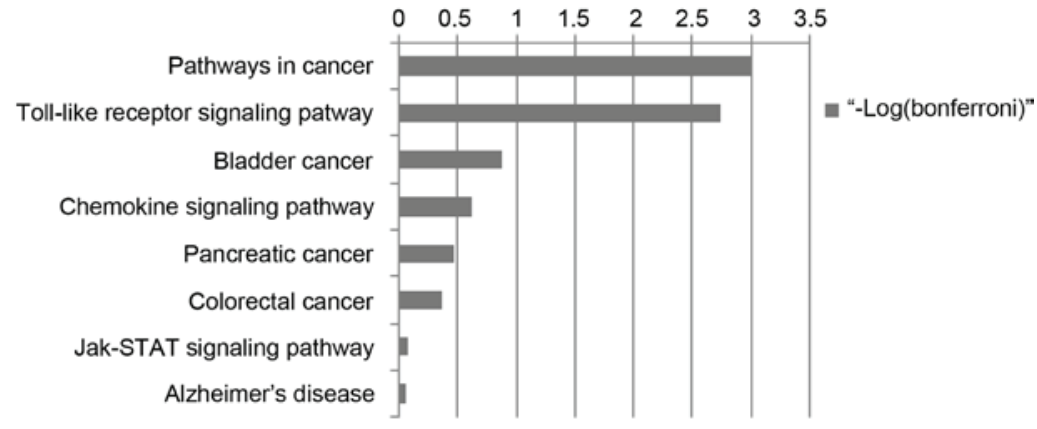

Figure 5. Kyoto Encyclopedia of Genes and Genomes pathway analysis demonstrates that the most significant pathway associated with psoriasis is the pathways in cancer, in addition to the Toll-like receptor signaling pathway. Jak-STAT, Janus kinase/signal transducers and activators of transcription.

In addition, the $E S R 1$ topological neighbors were selected from the interaction network; 56 pairs that directly interacted with the ESR1 gene are shown in Fig. 4, including nine top-ranked hub genes [APOE, BIRC5, cyclin D1 (CCDN1), FBJ murine osteosarcoma viral oncogene homolog (FOS), interleukin-8 $(I L-8)$, lipoprotein lipase (LPL), MMP9, STAT1, signal transducer and activator of transcription-3 (STAT3)] highlighted in red. The five genes (APOE, MCL1, COMP, $B I R C 5$ and $I L-1 B$ ) with anti-apoptotic function were directly or indirectly associated with the ESRI gene (Fig. 4). Three genes were indirectly linked with the $E S R I$ gene by binding to the ESRl gene topological neigbors, particularly by binding to the important apoptosis-regulating genes, such as B-cell CLL/lymphoma 2 (BCL2), MMP9 and CCND1.

Using KEGG pathway analysis (FDR $<0.05$ ), the pathways in cancer was detected to be most significantly associated with psoriasis, more so than the TLR signaling pathway, as shown in Fig. 5. Other pathways, including the chemokine signaling pathway and Janus kinase/signal transducers and activators of transcription signaling pathway were also detected in the present study; however, the significance was identified to be relatively lower. Seven of the top hub genes (FOS, CCNDI, IL-8, MMP9, $B I R C 5, S T A T 1$ and $S T A T 3$ ) were detected in response to pathways in cancer and five of the top hub genes [FOS, IL-8,IL-1B,STAT] and chemokine (C-X-C motif) ligand 10 (CXCL10)] exerted an effect on the TLR signaling pathway (data not shown). All of the above-mentioned top hub genes, apart from the CXCL10 gene, were involved in the first two pathways, demonstrating a direct association with the ESRI gene (Fig. 4). The present study demonstrated that four of the genes (MMP9, BIRC5, IL- $1 B$ and STATI) were highly associated with anti-apoptosis, regulation of apoptosis and programmed cell death.

\section{Discussion}

Psoriasis is a chronic inflammatory skin disorder. Significant decreases in keratinocyte apoptosis is a specific pathogenic phenomenon in psoriasis lesions compared with normal skin (3). In the present study, 927 DEGs were identified from the GEO database, GSE13355, which contained gene expression 
profiles for 58 psoriatic lesion samples and 64 normal skin samples. An interaction network with the 927 DEGs was then constructed (Fig. 2). Following PageRank analysis (Table I), ESRl and another 13 genes were selected as the top hub genes from 640 DEGs according to their PageRank score. In addition, the functions of the 14 top hub genes were analyzed by using GO term function analysis (Table II), indicating that the most significant enrichment function was associated with anti-apoptosis. Other functions, including response to organic substances, and regulation of apoptosis, programmed cell death and cell death, were also highly associated with psoriasis.

Apoptosis is a type of programmed cell death. Multiple factors control the extrinsic and intrinsic apoptotic signaling pathways. To maintain epidermal homeostasis, the complex apoptotic process is regulated by numerous anti-apoptotic molecules (5). The ESR1 gene, as well as five other top hub genes, has been demonstrated to exert an effect on anti-apoptosis in psoriasis according to the present results. Four of the genes (MCL1, COMP, BIRC5 and $I L-1 B)$ demonstrate an upregulated expression level in psoriasis. The other two genes (ESRI and APOE) demonstrate a downregulated level of expression. In addition to the six top hub genes, a further two highly expressed genes, MMP9 and STAT1, were identified to be involved in the regulation of apoptosis, programmed cell death and cell death. The MCL1 gene encodes an anti-apoptotic protein, myeloid cell leukemia-1. Ultraviolet B radiation therapy inhibits growth and induces apoptosis of human skin cells by downregulating $M C L 1$ expression (21). $I L-1 B$ induced IL-8 and p53 mRNA expression, as well as protein production of IL-8 (22). p53 overexpression has been described as a physiological reaction to hyperproliferation in order to resist keratinocyte apoptosis. Furthermore, knockdown of p53 expression levels in human keratinocytes accelerates MCL1 reduction, thereby enhancing apoptosis (23).

The ESRl gene encodes an ER $\alpha$ (24), which is a ligand-activated transcription factor that binds to estrogen (25). ER $\alpha$ is the receptor that transmits estrogen signaling; however, the function of $\mathrm{ER} \alpha$ in psoriasis development remains largely unknown. In the present study, the anti-apoptotic genes (BIRC5 and APOE) as topological neighbors, demonstrated a direct association with the ESRI gene (Fig. 4). Other anti-apoptotic genes (MCL1, IL-1B and COMP) demonstrated indirect connections with the ESR 1 gene by binding to the ESR1 topological neighbors, particularly by binding to the important apoptosis regulation genes, such as $B C L-2, M M P 9$ and CCND1 (Fig. 4). The MMP9 gene, an important gene in apoptosis regulation, has been demonstrated to be involved in the cell death pathways (26). Psoriasin (termed, S100 calcium-binding protein A7), which is highly expressed in tumors and hyperproliferative skin, enhances anti-apoptotic nuclear factor $-\kappa \mathrm{B}(\mathrm{NF}-\kappa \mathrm{B})$-mediated MMP-9 secretion in $\mathrm{ER} \alpha^{-}$breast cancer cells, but has the opposite effect in $\mathrm{ER} \alpha^{+}$ cells (27,28). CCDN1 and BCL2, at lower expression levels, were also associated with the ESRl gene (Fig. 4; Table I). A previous study regarding colorectal cancer demonstrated that $17 \beta$-estradiol activates p53 to inhibit cell proliferation by inhibiting CCDN1 (29). In addition, various studies have reported that a lower expression level of B-cell lymphoma 2 (Bcl-2) was strongly associated with psoriasis (4,30-32). The strong bcl-2-like protein 4 (BAX) expression, as well as the concomitant decrease in the level of $\mathrm{Bcl}-2$ expression indicated a resistance to BAX-mediated apoptosis in psoriasis (30). Thus, the reduced expression level of ESRI may lead to the upregulated expression of anti-apoptotic genes via mediation of the genes that are associated with apoptosis regulation, such as MMP9, BCL2 and CCND1. This provides evidence that the ESRl gene may be implicated in psoriatic apoptotic resistance and that ER $\alpha$ may exert a protective role against psoriatic proliferation. To the best of our knowledge, the underlying mechanism of the association between ESRI and the anti-apoptosis signaling pathway in psoriasis has not been fully elucidated; however, the present findings indicate that the reduced level of ESRI gene expression associated with psoriasis, is highly correlated with apoptosis resistance.

Using KEGG pathway analysis for the top 14 hub genes, the signaling pathway most significantly linked with psoriasis is the pathways in cancer, as well as the TLR signaling pathway (Fig. 5). As a classical pathway in psoriasis, the TRL signaling pathway has been shown to be important in psoriasis and is involved in detecting invading micro-organisms and producing pro-inflammatory stimuli to the skin (7). TLRs drive aberrant activation and unrestricted inflammatory responses (33).

In the current study, it is the first time, to the best of our knowledge, that the pathways in cancer has been detected to have the most significant association with psoriasis, more so than the TLR signaling pathway. Pathways in cancer exerts an effect on tumor proliferation by protecting tumor cells from cytokine-induced apoptosis. A previous study demonstrated that the thickened psoriatic skin displays a particular resistance of keratinocytes to interferon- $\gamma$ (INF- $\gamma$ )- and tumor necrosis factor- $\alpha$ (TNF- $\alpha)$-induced apoptosis by sustaining the activation of the phosphatidylinositol 3-kinase/Akt signaling pathway and consequently activating the anti-apoptotic NF- $\kappa \mathrm{B}$ cascade (4). Thus, the mechanisms of cell proliferation result in activation of anti-apoptotic molecules, which contribute to suppressing cytokine-induced apoptosis and maintaining the response to pro-inflammatory stimuli in keratinocytes (34). Hsu et al (35) reported that ER $\alpha$ inhibited bladder cancer cell growth by inhibiting Akt activity (35). Therefore, the present study hypothesized that ER $\alpha$ expression may activate INF- $\gamma$ and TNF- $\alpha$-induced apoptosis in cells by inhibiting Akt activity in psoriasis and various types of cancer, including bladder cancer.

The current study demonstrated that seven of the top hub genes that were involved in the pathways in cancer were directly associated with the ESRI gene. The above-mentioned evidence suggests that activating the pathways in cancer may protect the psoriatic cell from cytokine-induced apoptosis by inhibiting ER $\alpha$ expression. Further investigations are required to establish why the pathways in cancer is more significant than the cytokine-related TLR signaling pathway in apoptosis resistance in psoriasis.

In conclusion, activating the pathways in cancer, as well as reducing ER $\alpha$ expression promotes proliferation and resistance to apoptosis. The present results indicate that a lower $\mathrm{ER} \alpha$ expression level may induce psoriatic keratinocyte proliferation and anti-apoptosis effects via anti-apoptotic genes modulated by activation of the pathways in cancer. However, the contribution of individual factors requires elucidation. Future studies are required to investigate the functions of ER $\alpha$, 
the association between anti-apoptosis genes in psoriasis and whether these genes are involved in the biological mechanisms underlying the coordination of psoriatic keratinocyte apoptosis resistance.

\section{Acknowledgements}

The authors would like to thank Wilson Liao (Assistant Professor of Dermatology; Department of Dermatology, University of California, San Francisco, CA, USA) for providing the information processing methods. The present study was supported by grants from the National Natural Science Foundation of China (grant no. 30973758).

\section{References}

1. Christophers E: Psoriasis-epidemiology and clinical spectrum. Clin Exp Dermatol 26: 314-320, 2001.

2. Nestle FO, Kaplan DH and Barker J: Psoriasis. N Engl J Med 361 496-509, 2009

3. Laporte M, Galand P, Fokan D, de Graef C and Heenen M: Apoptosis in established and healing psoriasis. Dermatology 200: 314-316, 2000.

4. Madonna S, Scarponi C, Pallotta S, Cavani A and Albanesi C: Anti-apoptotic effects of suppressor of cytokine signaling 3 and 1 in psoriasis. Cell Death Dis 3: e334, 2012.

5. Portt L, Norman G, Clapp C, Greenwood M and Greenwood MT: Anti-apoptosis and cell survival: A review. Biochim Biophys Acta 1813: 238-259, 2011.

6. Sanchez MI, Shearwood AM, Chia T, Davies SM, Rackham O and Filipovska A: Estrogen-mediated regulation of mitochondrial gene expression. Mol Endocrinol 29: 14-27, 2015.

7. Miller LS: Toll-like receptors in skin. Adv Dermatol 24: 71-87, 2008.

8. Nair RP, Duffin KC, Helms C, Ding J, Stuart PE, Goldgar D, Gudjonsson JE, Li Y, Tejasvi T, Feng BJ, et al: Genome-wide scan reveals association of psoriasis with IL-23 and NF-kappaB pathways. Nat Genet 41: 199-204, 2009.

9. Swindell WR, Johnston A, Carbajal S, Han G, Wohn C, Lu J, Xing X, Nair RP, Voorhees JJ, Elder JT, et al: Genome-wide expression profiling of five mouse models identifies similarities and differences with human psoriasis. PloS One 6: e18266, 2011

10. Kerr MK: Linear models for microarray data analysis: Hidden similarities and differences. J Computat Biol 10: 891-901, 2003.

11. Bhat-Nakshatri P, Song EK, Collins NR, Uversky VN, Dunker AK, O'Malley BW, Geistlinger TR, Carroll JS, Brown M and Nakshatri H: Interplay between estrogen receptor and AKT in estradiol-induced alternative splicing. BMC Med Genomics 6: 21, 2013.

12. Franceschini A, Szklarczyk D, Frankild S, Kuhn M, Simonovic M, Roth A, Lin J, Minguez P, Bork P, von Mering C and Jensen LJ: STRING v9.1: Protein-protein interaction networks, with increased coverage and integration. Nucleic Acids Res 41 (Database Issue): D808-D815, 2013.

13. Snel B, Lehmann G, Bork P and Huynen MA: STRING: A web-server to retrieve and display the repeatedly occurring neighbourhood of a gene. Nucleic Acids Res 28: 3442-3444, 2000.

14. Page L, Brin S, Motwani R and Winograd T: The Page Rank citation ranking: Bringing order to the web. Stanford Info Lab, 1999.

15. Dellavalle RP, Schilling LM, Rodriguez MA, Van de Sompel H and Bollen J: Refining dermatology journal impact factors using Page Rank. J Am Acad Dermatol 57: 116-119, 2007.

16. Griffiths TL, Steyvers M and Firl A: Google and the mind: Predicting fluency with Page Rank. Psychol Sci 18: 1069-1076, 2007.
17. Bánky D, Iván G and Grolmusz V: Equal opportunity for low-degree network nodes: A Page Rank-based method for protein target identification in metabolic graphs. PloS One 8: e54204, 2013

18. Huang DW, Sherman BT and Lempicki RA: Systematic and integrative analysis of large gene lists using DAVID bioinformatics resources. Nat Protoc 4: 44-57, 2009.

19. Huang DW, Sherman BT and Lempicki RA: Bioinformatics enrichment tools: Paths toward the comprehensive functional analysis of large gene lists. Nucleic Acids Res 37: 1-13, 2009.

20. Albert R and Barabási AL. Statistical mechanics of complex networks. Rev Mod Phys 74: 47-97, 2002.

21. Park YK and Jang BC: UVB-induced anti-survival and pro-apoptotic effects on $\mathrm{HaCaT}$ human keratinocytes via caspase- and PKC-dependent downregulation of PKB, HIAP-1, Mcl-1, XIAP and ER stress. Int J Mol Med 33: 695-702, 2014

22. Rasmussen MK, Iversen L, Johansen C, Finnemann J, Olsen LS, Kragballe K and Gesser B: IL-8 and p53 are inversely regulated through JNK, p38 and NF-kappaB p65 in HepG2 cells during an inflammatory response. Inflamm Res 57: 329-339, 2008.

23. Chaturvedi V, Sitailo LA, Qin JZ, Bodner B, Denning MF, Curry J, Zhang W, Brash D and Nickoloff BJ: Knockdown of p53 levels in human keratinocytes accelerates Mcl-1 and Bcl-x $(\mathrm{L})$ reduction thereby enhancing UV-light induced apoptosis. Oncogene 24: 5299-5312, 2005.

24. Zang YC, Halder JB, Hong J, Rivera VM and Zhang JZ: Regulatory effects of estriol on $\mathrm{T}$ cell migration and cytokine profile: Inhibition of transcription factor NF-kappa B. J Neuroimmunol 124: 106-114, 2002.

25. Chang KC, Wang Y, Oh IG, Jenkins S, Freedman LP, Thompson CC, Chung JH and Nagpal S: Estrogen receptor beta is a novel therapeutic target for photoaging. Mol Pharmacol 77: 744-750, 2010.

26. Paolillo N, Piccirilli S, Giardina E, Rispoli V, Colica C and Nisticò S: Effects of paraquat and capsaicin on the expression of genes related to inflammatory, immune responses and cell death in immortalized human HaCat keratinocytes. Int J Immunopathol Pharmacol 24: 861-868, 2011.

27. Petersson S, Bylander A, Yhr M and Enerback C: S100A7 (Psoriasin), highly expressed in ductal carcinoma in situ (DCIS), is regulated by IFN-gamma in mammary epithelial cells. BMC Cancer 7: 205, 2007.

28. Sneh A, Deol YS, Ganju A, Shilo K, Rosol TJ, Nasser MW and Ganju RK: Differential role of psoriasin (S100A7) in estrogen receptor alpha positive and negative breast cancer cells occur through actin remodeling. Breast Cancer Res Treat 138: 727-739, 2013.

29. Hsu HH, Kuo WW, Ju DT, Yeh YL, Tu CC, Tsai YL, Shen CY, Chang SH, Chung LC and Huang CY: Estradiol agonists inhibit human LoVo colorectal-cancer cell proliferation and migration through p53. World J Gastroenterol 20: 16665-16673, 2014.

30. Koçak M, Bozdogan O, Erkek E, Atasoy P and Birol A: Examination of Bcl-2, Bcl-X and bax protein expression in psoriasis. Int J Dermatol 42: 789-793, 2003.

31. Gabr SA, Berika MY and Alghadir AH: Apoptosis and clinical severity in patients with psoriasis and $\mathrm{HCV}$ infection. Indian J Dermatol 59: 230-236, 2014.

32. Gündüz K, Demireli P, Vatansever S and Inanir I: Examination of bcl-2 and p53 expressions and apoptotic index by TUNEL method in psoriasis. J Cutan Pathol 33: 788-792, 2006

33. Liu Y, Yin H, Zhao M and Lu Q: TLR2 and TLR4 in autoimmune diseases: A comprehensive review. Clin Rev Allergy Immunol 47: 136-147, 2014.

34. Dallaglio K, Marconi A and Pincelli C: Survivin: A dual player in healthy and diseased skin. J Invest Dermatol 132: 18-27, 2012.

35. Hsu I, Yeh CR, Slavin S, Miyamoto H, Netto GJ, Tsai YC, Muyan M, Wu XR, Messing EM, Guancial EA and Yeh S: Estrogen receptor alpha prevents bladder cancer via INPP4B inhibited akt pathway in vitro and in vivo. Oncotarget 5: 7917-7935, 2014. 